\title{
Parameter Identification of a Shell Transfer Arm Using PSO and Similarity Degree Analysis
}

\author{
Qiang-Qiang Zhao and Bao-Lin Hou
}

\begin{abstract}
A shell transfer arm is a complex mechanical electro-hydraulic system with multiple parameters. An accurate dynamic model is the precondition of improved design. However, during the modeling process, several parameters that have a significant effect on the performance of the system can be achieved only by identification methods because they are hard to measure. According to the structure and control theory of the shell transfer arm, the analytical model and control model are built. To evaluate the level of similarity of the built model and real system, the numerical similarity and shape similarity of the angular velocity of the arm are calculated, and the combination similarity of numerical similarity and shape similarity is set as the fitness function of Particle Swarm Optimization (PSO). Four unknown parameters are identified by PSO. The identification results with respect to the simulation data show that the identification accuracy of PSO can acquires the requirement. And the identification results with respect to the test data show that the built model is accurate, and parameter identification of the shell transfer arm based on PSO and similarity degree analysis of time-vary data is feasible and effective.
\end{abstract}

Index Terms-Shell transfer arm, dynamic modeling, parameter identification, particle swarm optimization, similarity degree analysis.

\section{INTRODUCTION}

A shell transfer arm is an important component of the ammunition automatic loader system of a large caliber self-propelled cannon. It is mainly used to transport the shell pulled out from the magazine to a required position [1]. The reliability of the whole ammunition automatic loader system is severely reduced because the location accuracy of the shell transfer arm is often out of tolerance during its service time. Therefore, an improved design for the shell transfer arm is imperative. Building an accurate dynamic model of the shell transfer arm is of great significance for deducing how the system characteristics of the arm change during its running processes, for making suggestions for an improved design, and even for achieving the fault diagnosis and fault prediction. A core problem of mathematical modeling is to determine the key parameters. However, some key parameters of the shell transfer arm can be achieved only by

Manuscript received October 26, 2016; revised December 23, 2016. This work was supported in part by the Natural Science Foundation of China under Grant 51175266, and the Important National Basic Research Program of China (973 Program) under Grant 6132490102.

Q. Q. Zhao is with School of Mechanical Engineering, Nanjing University of Science and Technology, Nanjing, 210094 China (e-mail: zqqlzl@139.com)

B. L. Hou is with School of Mechanical Engineering, Nanjing University of Science and Technology, Nanjing, 210094 China (e-mail: houbl@njust.edu.cn). identification methods because they are unable or hard to measure.

Nowadays, the modern system parameter identification method includes set membership identification method, neural network identification method, fuzzy logic identification method, and intelligent algorithm identification method [2]. The intelligent algorithm identification method is widely used in parameter identification of electromechanical system. Intelligent algorithm mainly includes genetic algorithm, ant colony algorithm, differential evolution algorithm, and particle swarm optimization (PSO). Due to its simple arithmetic, fewer parameters, easy to implement, and good optimizing capacity, PSO shows a good performance in continuous optimization problem and discrete optimization problem, and it has been a research hotspot of intelligent optimization field [3]. Bingül [4] identified the inertial parameters of a RX-60 robot by PSO, and compared the performance of PSO and least square method. Wang [5] used PSO to determine the parameters of spindle-holder and holder-tool interfaces. Liu [6] achieved the parameter identification of a high-power permanent magnet synchronous motor for stator resistance and load torque by PSO. And Abdullah [7] presents a transfer function model prediction with PSO.

However, it need a suitable fitness function to achieve the parameter identification by PSO. For electromechanical system, in general, the error sum of squares of real outputs and prediction outputs is set as the fitness function ${ }^{[8-9]}$. However, when the outputs of the system are time-varying data, it is not enough to evaluate the comparability of the simulation model and real system only by the error sum of squares. Therefore, the similarity degree of the time-varying data is presented to evaluate the comparability of the systems, and is set as the fitness function of PSO. Finally, the initial pressure of the accumulator, the sensitivity of the tachometer generator, transmission efficiency of the worm and worm gear, and the polytropic exponent of the gas of the shell transfer arm are identified to prove that the presented method is feasible and effective.

\section{MODELING OF THE SHELl TRANSFER ARM}

\section{A. Dynamic Modeling}

As shown in Fig.1, the shell transfer arm is driven by two parallel series motors. The reduction box consists of a two-stage spur gear transmission and a one-stage worm and worm gear transmission. The worm gear is installed on the trunnion of the cannon. The arm is fixed with the worm gear, and it can rotate around the trunnion. The balance machine consists of a cylinder and an accumulators, and it is used to balance the gravitational torque of the arm to reduce the load 
of the motors. An angular displacement sensor and an angular velocity sensor (tachometer generator) are installed on the arm to provide the angle signal to rotate the shell transfer arm to a required position [1].

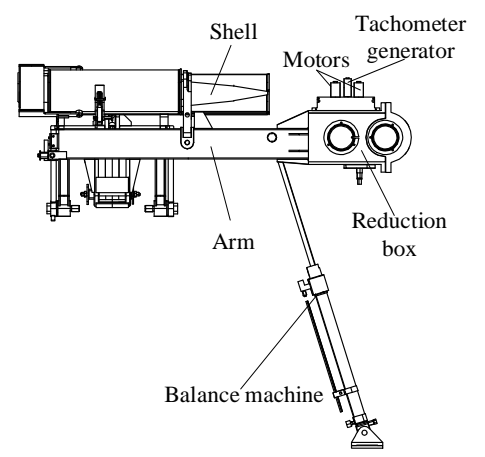

Fig. 1. The structure of the shell transfer arm.

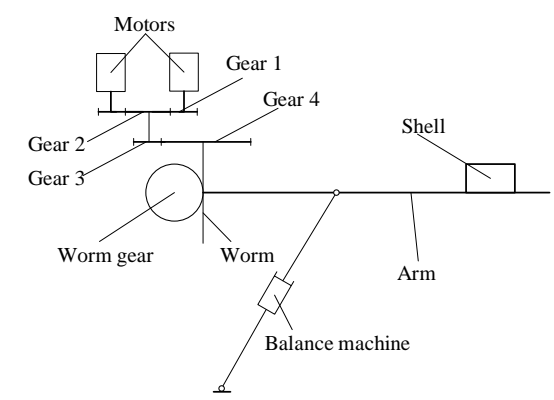

Fig. 2. The working principle of the shell transfer arm.

Fig. 2 shows the working principle of the shell transfer arm. It is a typical single degree of freedom system, and according to its force analysis, the dynamic equations of the arm can be achieved:

$$
J \ddot{\theta}=Q
$$

where $\theta$ is the rotation angle of the arm, $Q$ is the equivalent torque that acts on the arm, and $J$ is the rotational inertia of the system.

$$
\begin{gathered}
J=2 I_{01} i_{1}^{2} \eta_{1} \eta_{2} \eta_{3}+I_{23} i_{2}^{2} \eta_{2} \eta_{3}+I_{45} i_{3}^{2} \eta_{3}+I_{6} \\
Q=2 T_{d} i_{1} \eta_{1} \eta_{2} \eta_{3}-T_{R}+T_{G}
\end{gathered}
$$

where, $i_{1}$ is the total transmission ratio of the system; $i_{2}$ is the transmission ratio from gear 3 to worm gear; $i_{3}$ is the transmission ratio of the worm and worm gear. $\eta_{1}, \eta_{2}$, and $\eta_{3}$ are the transmission efficiency between gear 1 and gear 2 , gear 3 and gear 4 , worm and worm gear respectively. $I_{01}$ $I_{23}, I_{45}$, and $I_{6}$ are the equivalent rotational inertia of motor and gear1, gear 2 and gear 3, gear 4 and worm gear, and worm, the arm and the shell, respectively. $T_{d}$ is the driving torque of the motor. $T_{R}$ is the support torque of the balance machine that acts on the arm. $T_{G}$ is the gravitational torque of the arm and the shell.

$T_{G}$ depends on the rotation angle $\theta$ of the arm:

$$
T_{G}=L m g \cos (\theta+\alpha)
$$

where, $L$ is the distance from the center of mass of the arm and shell to the rotation center of the arm. $m$ is the mass of the arm and shell. $\alpha$ is the angle between the line from the center of mass of the arm and shell to the rotation center of the arm and horizontal line when the shell transfer arm is at the horizontal.

$T_{R}$ depends on the pressure of the cylinder and the rotation angle $\theta$ of the arm, and the pressure of the cylinder $p$ :

$$
p=p_{0} S\left(\frac{V_{0}}{V_{0}-\Delta V}\right)^{n}
$$

where $p_{0}$ is the initial pressure of the accumulator; $S$ is the area of the piston of the cylinder; $V_{0}$ is the initial volume of the gas; $\Delta V$ is the change volume of the gas; and $n$ is the polytropic exponent of the gas.

Then, the torque of the balance machine acts on the arm can be written as:

$$
T_{R}=l p_{0} S\left(\frac{V_{0}}{V_{0}-\Delta L S}\right)^{n}
$$

where $l$ is the distance from the rotation center of the arm to balance machine, and $\Delta L$ is the moving distance of the piston.

\section{B. Control System Modeling}

The shell transfer arm is driven by two parallel series motors, and dynamic equation are as follows [10]:

$$
\left\{\begin{array}{c}
U=E+R I+L \frac{d I}{d t} \\
\Phi=K I \\
E=C_{e} \Phi \omega \\
T=C_{T} \Phi I
\end{array}\right.
$$

where, $U$ is the voltage of the motors; $E$ is the back electromotive force; $R$ is the resistance of the motors; $I$ is the armature current; $L$ is the electric inductance; $\Phi$ is the magnetic flux; $K$ is the excitation coefficient; $C_{e}$ is the back electromotive force coefficient; $C_{T}$ is the electromagnetic torque coefficient; $\omega$ is the rotation speed of the motor; and $T$ is the output torque of the motor.

The control system of the shell transfer arm has no request on transient characteristics, and only the system' up-time and location accuracy are required. Therefore, few parameters need to be considered during the designing process of the control system. The motion process of the shell transfer arm is shown in Fig.3. AB is the motion range of the arm. When the arm rotates from point $C$ to point $D$ as required, the whole motion process can be divide into two stages. In stage CE, it only needs to control the rotation direction of the motors, which means it just needs to apply a rated voltage on the motors to cause the motors to rotate at the rated speed to drive the arm rotate to point $\mathrm{D}$ at full speed. When the arm rotates 
to point $\mathrm{E}$, the control system becomes a closed-loop position system, and the arm is located at point D accurately. The motion speed curve of the arm is shown in Fig. 4.

The driving circuit controls the speed of the motors by changing the average voltage applying for the motors. The voltage is a quasi-pulse which is similar to pulse width modulation. The motors have two opposite direction field windings for controlling the rotation direction. The power device is the insulated gate bipolar transistor (IGBT) for simplifying the driving circuit. The control signal is generated by a closed-loop proportional-derivative controller. There is a threshold circuit in the trigger circuit to prevent the two field windings from being activated at same time.

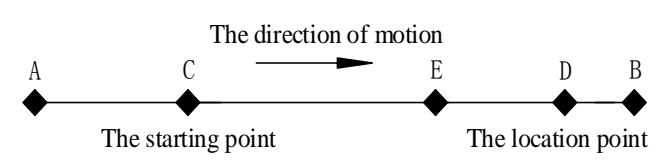

Fig. 3. The sketch diagram of the motion process of the arm.

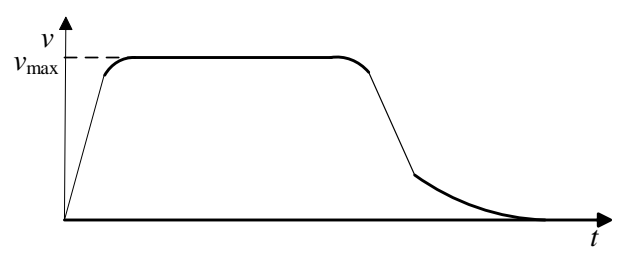

Fig. 4. The angular velocity of the shell transfer arm.

Based on the analysis hereinabove, the dynamic model and control model built in Simulink are shown in Fig.5 and Fig.6.

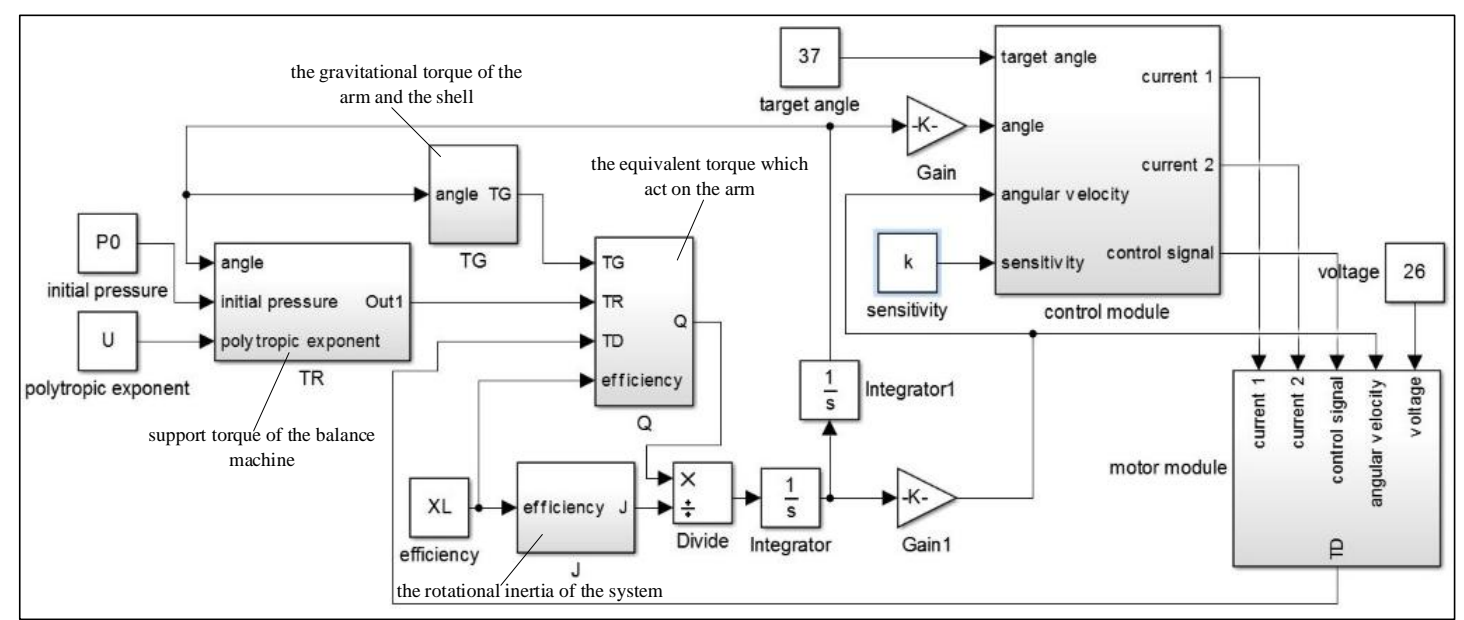

Fig. 5. The dynamic model of the shell transfer arm built in Simulink.

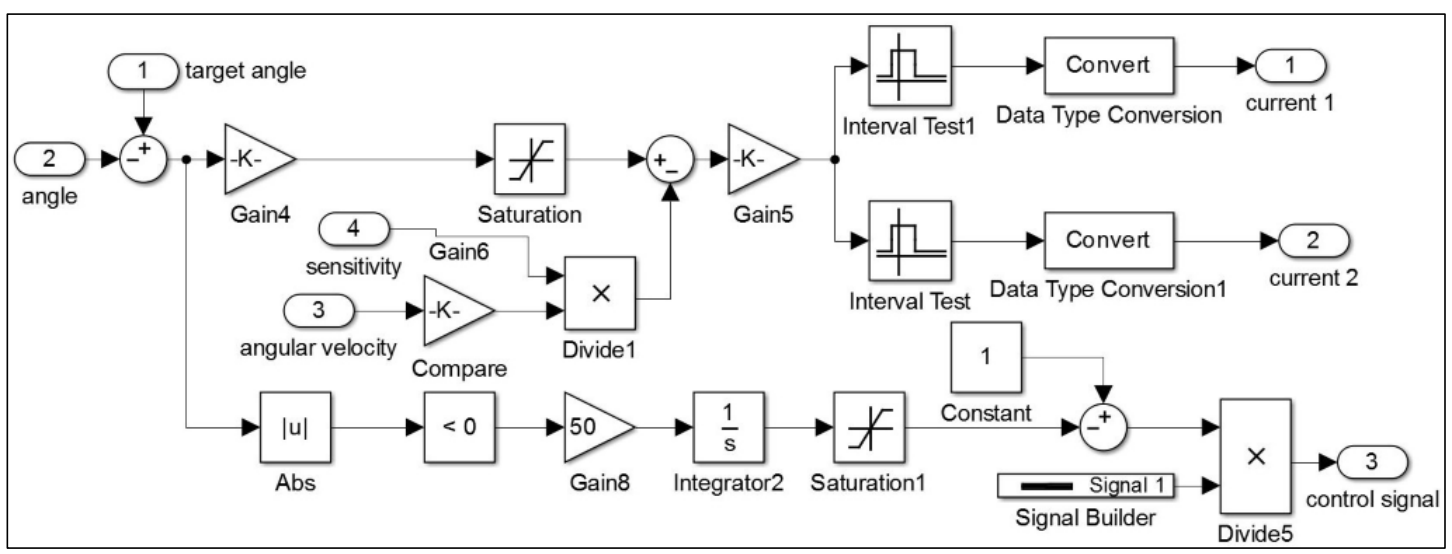

Fig. 6. The control module of the shell transfer arm.

\section{The Key Parameters Need to Be Identification}

Many parameters are included in the dynamic model and control model of the shell transfer arm. Some of the parameters are certainty, and can be achieved by calculational methods or measurement methods, such as the equivalent rotational $I_{01}, I_{23}, I_{45}, I_{6}$. However, some of the parameters are unknown and hard to measure. And this type of parameter can be obtained by identification methods. The four parameters need to be identified included in the models are as follows:

(1) The initial pressure of the accumulator $p_{0}$.
The power of the balance machine is provided by a bladder accumulator. The pressure of the cylinder will change if $p_{0}$ changes. Therefore, the shell transfer arm will be unstable if the pressure of the cylinder changes.

(2) The sensitivity of the tachometer generator $k$.

The location accuracy is directly influenced by the sensitivity of the tachometer generator. $k$ may be abnormal because of the vibration when the gun is shooting, the temperature changes, or there is electromagnetic interference.

(3) The transmission efficiency of the worm and worm gear $\eta_{3}$. 
$\eta_{3}$ depends on relative sliding velocity of the tooth surface, and general the relative sliding velocity of the tooth surface is unknown.

(4) The polytropic exponent of the gas $n$.

$n$ is a uncertain value during the accumulator's pressurized process. The oil reserve of the accumulator will be obvious changed if $n$ changes.

\section{IDENTIFICATION METHOD BASED ON PSO AND SIMILARITY DEGREE ANALYSIS}

\section{A. PSO Algorithm}

PSO algorithm is an evolutionary computation technique that was proposed by Eberhart and Kennedy ${ }^{[11]}$. The core thought of PSO is to update the speed and position of the particle by tracking the current individual optimum value and group optimum value, and set the group optimum as the optimal solution when iteration satisfied the stopping criterion. PSO updates the speed and position of the particles according to equation (8) and equation (9):

$$
\begin{gathered}
V_{i d}^{t+1}=\sigma V_{i d}^{t}+c_{1} r_{1}\left(P_{i d}^{t}-X_{i d}^{t}\right)+c_{2} r_{2}\left(P_{g d}^{t}-X_{i d}^{t}\right) \\
X_{i d}^{t+1}=X_{i d}^{t}+V_{i d}^{t+1}
\end{gathered}
$$

where, $\sigma$ is the inertia weight, $d=1,2, \ldots, D, i=1,2, \ldots, n$, $t$ is the current iteration time, $V_{i d}$ and $X_{i d}$ are the speed and position of the particle, respectively. $P_{i d}$ and $P_{g d}$ are the individual optimum value and group optimum value, respectively. $c_{1}$ and $c_{2}$ are acceleration factors, and they are nonnegative constant. $r_{1}$ and $r_{2}$ are random numbers in the range [0 1$]$. The speed is usually limited to the range $\left[-X_{\max }, X_{\max }\right]$ to prevent a blind search.

\section{B. Similarity Degree Analysis}

For the shell transfer arm, the measurable response variable and the output of the simulation model are all the angular velocity of the arm, and they are all time-vary data. Therefore, the key to evaluate the accuracy of the identification results is calculating the comparability of the two time-vary data. The comparability size is expressed by similarity degree $S$. The range of $S$ is $[0,1]$. $S=0$ denotes that the two systems are completely alien; $S=1$ denotes that the two systems are identical; and $0<S<1$ denotes the level of similarity of the two systems ${ }^{[12]}$.

For two time-vary data $X=\left[x_{1}, x_{2}, \ldots, x_{n}\right]$ and $X^{\prime}=\left[x_{1}^{\prime}, x_{2}^{\prime}, \ldots, x_{n}^{\prime}\right]$, the similarity degree $S$ of the two time-vary data is comprised of the numerical similarity degree $S_{1}$ and the shape similarity degree $S_{2}$. According to multi-attribute and multi-index theory ${ }^{[13]}$, the similarity degree $S$ is better to be calculated by multiplication than addition. Hence, $S$ can be written as:

$$
S=\sqrt{S_{1} S_{2}}
$$

The numerical similarity $S_{1}$ is expressed by the weighted average of relative error, the numerical similarity of each point $S_{i}$ is:

$$
s_{i}=\left\{\begin{array}{c}
1-\frac{\left|x_{i}-x_{i}^{\prime}\right|}{\left|x_{i}\right|}, x_{i} x_{i}^{\prime}>0 \\
0, \quad x_{i} x_{i}^{\prime}<0 \quad i=1,2, \ldots, n \\
0, x_{i}=0 \text { or } x_{i}^{\prime}=0 \\
1, x_{i}=x_{i}^{\prime}=0
\end{array}\right.
$$

The weight of arbitrary point is expressed by the ratio of the point's value and the sum of the all points:

$$
\beta_{i}=\frac{\left|x_{i}\right|}{\sum_{i=1}^{n}\left|x_{i}\right|}
$$

Therefore the numerical similarity $S_{1}$ can be written as follows:

$$
S_{1}=\sum_{i=1}^{n} s_{i} \beta_{i},(i=1,2, \ldots, n)
$$

The shape similarity is calculated by landmark dividing method, the main process is as follows ${ }^{[14]}$ :

(1) Normalize the original time-varying data $X$ to obtain a new time-varying data $\bar{X}: \bar{x}_{i}=\left(x_{i}-x_{\min }\right) /\left(x_{\max }-x_{\min }\right)$. where $x_{\max }$ and $x_{\min }$ are the maximum value and minimum value of $X$, respectively.

(2) Subtract the adjacent elements of data $\bar{X}$ to obtain a new time-varying data $Y: y_{i}=\bar{x}_{i+1}-\bar{x}_{i}$.

(3) Multiply the adjacent elements of data $Y$ to obtain a new time-varying $Z: z_{i}=y_{i} y_{i+1}$.

(4) Determine the index value of the negative $p_{i}$ in data $Z$, and the index value of the local extremum in $\bar{X}$ is $p_{i}+1$. Then, Ignore the extremum points if the increase is less than $\varepsilon$ and the time difference is less than $\xi$, and the other extremum points are the landmark of data $\bar{X} . \varepsilon$ and $\xi$ are determined by the noise that needs to be filtered out.

(5) Assume that data $\bar{X}$ has $m$ landmarks. Therefore, $\bar{X}$ is divided into $m+1$ subintervals. Define the slope of each subinterval:

$$
k_{i}=\left(\bar{x}_{j+1}-\bar{x}_{j}\right) / \Delta t
$$

$(i=1,2, \ldots, m+1, j=1,2, \ldots, m)$, where $\bar{x}_{j}$ is the $j$ th landmark in $\bar{X}$, and $\Delta t$ is the time difference of the $j$ th landmark and $(j+1)$ th landmark.

Define that two subintervals of are $\bar{X}$ and $\bar{X}$, similar if the slope difference of the two subintervals is less than a given threshold, and the shape similarity is defined as the ratio of the length of the similar subintervals and the whole data. Therefore, $S_{2}$ can be expressed as: 


$$
S_{2}=\frac{\sum_{i=1}^{m} L\left(\bar{X}_{i}\right)+L\left(\bar{X}_{i}{ }_{i}\right)}{L(X)+L\left(X^{\prime}\right)}
$$

where $L(\bullet)$ denotes the length of the whole time-varying data, and $m$ is the number of the similar subintervals.

When $S_{1}$ and $S_{2}$ are carried out, the combination similarity degree $S$ can be obtained according to equation (10). And in this paper, $S$ is set as the fitness function of PSO to achieve the parameters identification of the shell transfer arm.

\section{System Testing AND PARAMETERS IDENTIFICATION}

\section{A. System Testing}

The angular velocity of the shell transfer arm needs to be tested before the identification process. The angular velocity is tested by a single-axis gyro CS-ARS-02 with measuring range $\pm 200 \%$ s. Its output voltage is $0 \mathrm{~V} \sim 5 \mathrm{~V}$, the resolution ratio is less than $0.15 \%$, and the sampling frequency is $1000 \mathrm{~Hz}$. As shown in Fig.9, the consistency of the test results are very well, which means that the test results are dependable.

It needs to note that during the shell transfer arm's location process, the angular velocity data of the shell transfer arm has an obvious vibration because of the vibration of the shell in the cradled tray. However, the shell in simulation model is without vibration. Therefore, only the first $700 \mathrm{~ms}$ of data are used to calculate the similarity degree of the time-varying data, and the data after $700 \mathrm{~ms}$ are ignored.

\section{B. Parameters Identification}

The parameters identification process are as follows:

1) Set the parameters of the PSO, and generate the potential solution randomly.

2) Call the Simulink procedure, and simulate with respect to each potential solution to obtain the angular velocity curve family of the shell transfer arm.

3) Calculate the similarity degree $S$ of the angular velocity curve from step (2) and test, and the similarity degree is the fitness function of PSO.

4) Determine the individual optimum value and group optimum value according to $S$ from step (3)

5) Update the position and speed of the particles according to equation (8) and equation (9), and repeat step (2).

6) Update the individual optimum values and group optimum values according to the fitness form step (3).

7) Repeat step (5) and step (6) until the fitness function satisfies the predetermined requirements or reaches the maximum iteration step. Then, output the final group optimum values, and the final group optimum values are the identification results.

During the identification process in this paper, the parameters of the PSO are as follows: the population size is 80 , the maximum iteration step is $150, c_{1}=1.2, c_{2}=1.7$, and the inertia weight $\sigma$ is a linearly decreasing weight, $\sigma(k)=\sigma_{\min }+\left(\sigma_{\max }-\sigma_{\min }\right)\left(T_{\max }-t\right) / T_{\max }$, where $n$ is the current iteration step, $T_{\max }$ is the maximum iteration step, and $\sigma_{\max }=0.9, \sigma_{\min }=0.4$. The range of the parameters need to be identified are as follows: the range of the initial pressure of the accumulator is [3.0 3.5] $\mathrm{MPa}$, the sensitivity of the tachometer generator is [0.6 1.1$]$, the transmission efficiency of worm and worm gear is [0.47 0.6], and the polytropic exponent of the gas is [1.1 1.5].

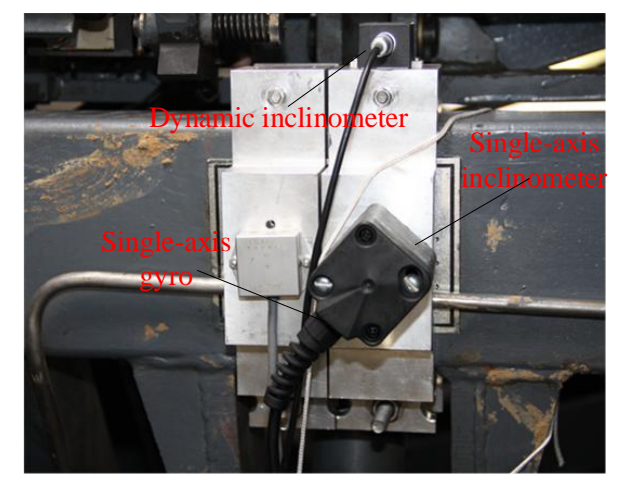

Fig. 8. The test picture.

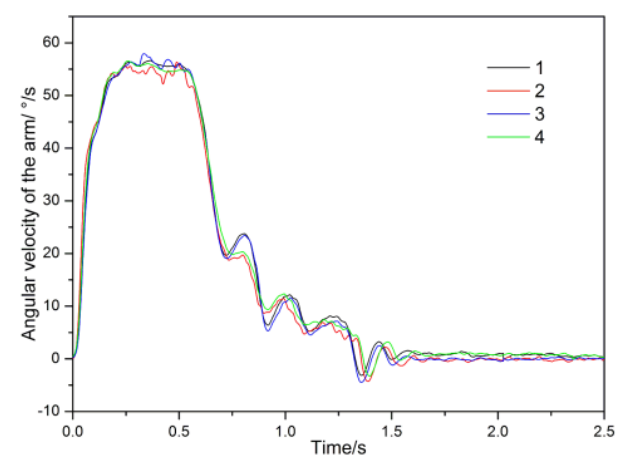

Fig. 9. Some of the test results.

Because the parameters need to be identified are hard or unable to measure, the identification accuracy is verified by the simulation data. Then the test data are used to identify the parameters of the real system.

The identification results of the simulation data are shown in Tab.1. The average relative error of the four parameters are $0.49 \%, 0.42 \%, 0.17 \%$, and $0.90 \%$. And which means that the identification accuracy satisfies the requirement.

The test data are used for parameters identification for 5 times, and the identification results are in shown in Tab.2. The average values of the identification for four parameters are $3.0459 \mathrm{MPa}, 0.9969,0.5531$, and 1.4941 , respectively. The standard deviations are $0.0147,0.0029,0.0110$, and 0.0066, respectively. The standard deviations and identification results show that the consistency of the identification results are very well, and the identification results are in a reasonable range.

Fig. 10 shows the comparison of the test data and the simulation data corresponding to the identification results. Fig.11 shows the optimization procedure of the similarity degree. The final similarity degree is 0.9752 , the high similarity degree shows that the simulation model built in this paper is accurate, and the parameters identification method based on PSO and similarity degree analysis is effective. 
TABLE I: THE IDENTIFICATION RESULTS OF SIMULATION DATA

\begin{tabular}{|c|c|c|c|c|c|c|c|}
\hline \multicolumn{2}{|c|}{ Initial pressure $P_{0} / \mathrm{MPa}$} & \multicolumn{2}{|c|}{ Sensitivity $k$} & \multicolumn{2}{|c|}{ transmission efficiency $\eta_{3}$} & \multicolumn{2}{|c|}{ polytropic exponent $n$} \\
\hline $\begin{array}{c}\text { Theoretical } \\
\text { Value }\end{array}$ & $\begin{array}{c}\text { Identification } \\
\text { value }\end{array}$ & $\begin{array}{c}\text { Theoretical } \\
\text { Value }\end{array}$ & $\begin{array}{c}\text { Identification } \\
\text { value }\end{array}$ & $\begin{array}{c}\text { Theoretical } \\
\text { Value }\end{array}$ & $\begin{array}{c}\text { Identification } \\
\text { value }\end{array}$ & $\begin{array}{c}\text { Theoretical } \\
\text { Value }\end{array}$ & $\begin{array}{c}\text { Identificatio } \\
\mathrm{n} \text { value }\end{array}$ \\
\hline 3.20 & 3.2114 & 1.00 & 1.0026 & 0.62 & 0.6213 & 1.00 & 1.0124 \\
\hline 3.10 & 3.0887 & 0.95 & 0.9518 & 0.60 & 0.6011 & 1.10 & 1.0915 \\
\hline 3.00 & 3.0321 & 0.90 & 0.8986 & 0.58 & 0.5786 & 1.20 & 1.2126 \\
\hline 2.90 & 2.9096 & 0.85 & 0.8587 & 0.55 & 0.5511 & 1.30 & 1.3111 \\
\hline 2.80 & 2.7912 & 0.80 & 0.8038 & 0.50 & 0.5001 & 1.40 & 1.4081 \\
\hline
\end{tabular}

TABLE II: THE IDENTIFICATION RESULTS OF TEST DATA

\begin{tabular}{ccccc}
\hline \hline & Initial pressure $P_{0} / \mathrm{MPa}$ & Sensitivity $k$ & Transmission efficiency $\eta_{3}$ & Polytropic exponent $n$ \\
\hline 1 & 3.0215 & 0.9973 & 0.5338 & 1.4986 \\
2 & 3.0641 & 0.9991 & 0.5662 & 1.4837 \\
3 & 3.0578 & 1.0000 & 0.5607 & 1.4888 \\
4 & 3.0438 & 0.9964 & 0.5526 & 1.4993 \\
5 & 3.0422 & 0.9916 & 0.5523 & 1.5000 \\
Mean value & 3.0459 & 0.9969 & 0.5531 & 1.4941 \\
Mean square error & 0.0147 & 0.0029 & 0.0110 & 0.0066 \\
\hline \hline
\end{tabular}

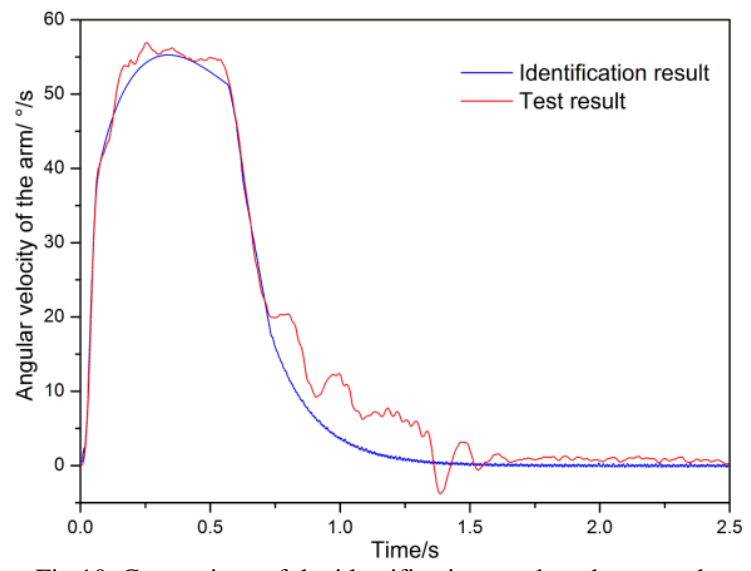

Fig.10. Comparison of the identification result and test result.

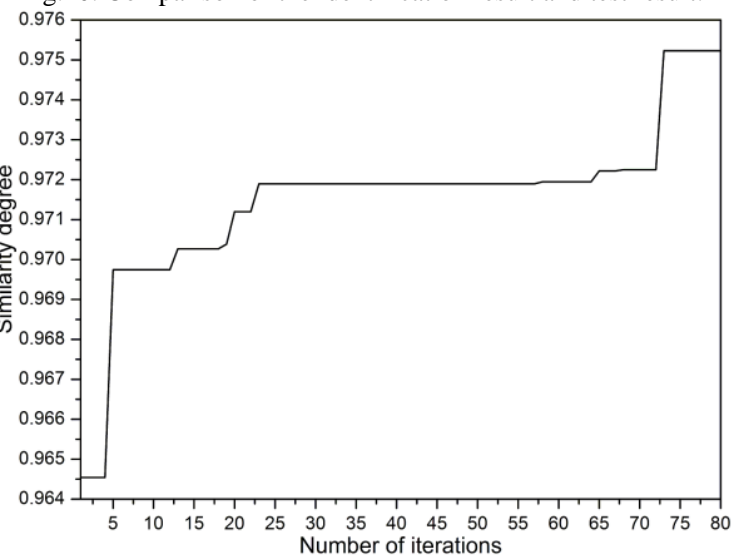

Fig. 11. The optimization process of the similarity degree.

\section{Conclusions}

In this paper, a parameter identification method using PSO and time-vary data similarity degree analysis for a shell transfer arm is presented, and the following conclusions are obtained:

1) A dynamic model and a control model are built according to the structure and control theory of the shell transfer arm, and the dynamic model and control model are solved in Simulink.

2) The similarity degree of the angular velocity of the arm is calculated for evaluating the level of similarity of the built model and real system. The similarity degree is set as the fitness function of PSO, and four parameters of the shell transfer arm are identified by
PSO. Due to the parameters are hard even unable to measured, the identification accuracy is verified by simulation data, and the feasibility of the method is verified by the test data.

3) The final identification results show that the dynamic model and control model built in this paper are accurate, and the parameters identification method using PSO and similarity degree analysis is effective.

\section{REFERENCES}

[1] B. L. Hou, J. M. Qiao, and C. M. Liu, Ammunition Automatic Loading System of Howitzer, 1st ed. Beijing: Publishing of Ordnance Industry, 2010, ch. 3, pp. 62-68.

[2] J. K. Liu, X. R. Shen, and L. Zhao, System Identification Theory and MATLAB Simulation, Beijing: Electronic Industry Press, 2013, ch. 1.

[3] W. B. Wang, "Research on particle swarm optimization algorithm and its application," M.S. thesis, Chengdu: Southwest Jiaotong University, Chengdu, China, 2012.

[4] Z. Bingül and O. Karahan. "Dynamic identification of staubli RX-60 robot using PSO and LS methods," Expert Systems with Applications, vol. 38, pp. 4136-4149, April 2011.

[5] E. H. Wang. B. Wu, Y. M. Hu, J. Wang, and S. Z. Yang, "Parametric identification of spindle-holder and holder-tool interfaces," Journal of Vibration and Shock, vol. 33, no. 10, pp. 50-54, May 2014.

[6] L. Liu, W. X. Liu, and S. A. Cartes, "Particle swarm optimization-based parameter identification applied to permanent magnet synchronous motors," Engineering Application of Artificial Intelligence, vol. 21, pp. 1092-1100, October 2008.

[7] M. A. Abdullah, S. Ahmad, and S. F. Toha, "Particle swarm optimization-based identification of a bouncing spherical robot," presented at the 10th Asian Control Conference, Kota Kinabalu: IEEE, pp. 1-6, June 2015.

[8] J. S. Hu, C. X. Guo, and Y. J. Cao. "Generator parameter identification based on extended particle swarm optimization method," Automation of Electric Power Systems, vol. 28, no. 6, pp. 35-40, March 2004.

[9] H. Ma, W. H. Li, X. X. Xiao, H. Liu, and Z. N. Jiang. "A new particle swarm optimization approach to the inertia parameters identification of on-orbit space robot," Journal of Astronautics, vol. 36, no. 3, 278-283, March 2015.

[10] H. W. Li, "Characteristic simulation and analysis of series motor," Micromotors, vol.43, no. 8, pp. 95-97, August 2010.

[11] J. Kennedy, and R. Eberhart. "Particle swarm optimization," presented at IEEE International Conference on Neural Network, Perth, Australia: IEEE, pp. 1942-1948, November 1995.

[12] J. He, "Research on validity evaluation and parameter calibration of generator model based on hybrid dynamic simulation," M.S. thesis, Chongqing: Chongqing University, Chongqing, China, 2011.

[13] Z. Xu, Uncertain multi-attribute making methods and application. Springer, 2015, ch.2.

[14] Y. L. Yang, J. Q. Qin, C. C. Di, Y. H. Deng, and X. G. Zhang, "Modeling and firing simulation accuracy assessment of a gun-power-recoil test table," Journal of Vibration and Shock, vol. 33, no. 10, pp. 127-130, May 2014. 


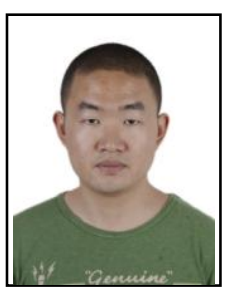

Q. Q. Zhao was born in China in 1987. He received his bachelor in mechanical engineering at Nanjing University of Science and Technology, Nanjing, China, 2006.

He received his master in mechanical engineering at Nanjing University of Science and Technology, Nanjing, China, 2010.

Currently, he is a Ph.D. at Nanjing University of dynamic modeling and fault diagnosis of electromechanical system.

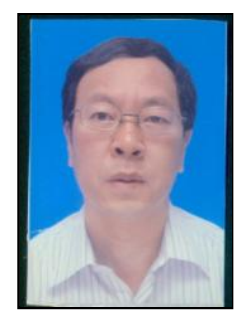

B. L. Hou was born in China in 1965 . He is currently a professor at School of Mechanical Engineering, Nanjing University of Science and Technology, China. He received his $\mathrm{PhD}$ degree on from Nanjing University of Science and Technology. His research interests include ammunition automatic loading system and magneto rheological shock absorber. 